\title{
Impact of intrinsic backbone chain stiffness on the morphologies of bottle-brush diblock copolymers
}

\author{
Alexandros Chremos ${ }^{\mathrm{a}, *}$, Panagiotis. E. Theodorakis ${ }^{\mathrm{b}, *}$ \\ ${ }^{a}$ Materials Science and Engineering Division, National Institute of Standards and \\ Technology, Gaithersburg, MD, 20899, USA \\ ${ }^{b}$ Institute of Physics, Polish Academy of Sciences, Al. Lotników 32/46, 02-668 Warsaw, \\ Poland
}

\section{Abstract}

Self-assembly methods are used for the production of photonic and nanoporous materials derived from block copolymers. In this context, bottle-brush copolymers have demonstrated a number of advantages over the respective linear copolymers, such as faster self-assembly kinetics and a richer morphology behavior. However, the effect of intrinsic molecular stiffness on the morphology of bottle-brush copolymers has been previously overlooked. Here, we investigate the role of the intrinsic backbone chain stiffness on the morphology behavior of bottle-brush diblock copolymers by using molecular dynamics simulations of a bead-spring coarse-grained model. We focus on bottle-brush macromolecules having blocks of the same volume fraction and asymmetricin-length side chains. We find that an increase of the backbone stiffness triggers an order-order transition from hexagonal packed cylinders to lamellar morphologies with asymmetric domain spacings, which is of particular

\footnotetext{
*Corresponding author

Email addresses: alexandros.chremos@nist.gov (Alexandros Chremos), panos@ifpan.edu.pl (Panagiotis. E. Theodorakis)
} 
interest for the manufacturing of nanopatterning and semiconductor applications. The change in the morphology is due to the effective many-body attractions between the blocks resulting in a parallel stacking that disrupts the symmetry of the cylindrical morphology. We anticipate that our work will underline the significance of intrinsic molecular stiffness in the self-assembly of polymer systems, which has been previously neglected.

Keywords: Instrinsic backbone stiffness, Self-assembly, Bottle-brush copolymers, Molecular Dynamics simulations, Bead-spring model

\section{Introduction}

The self-assembly of block copolymers has been a powerful tool for manufacturing nanostructured materials.[1, 13, 27] However, there are many difficulties in controlling the topology, domain spacing, and alignment of the self-assembled patterns of these materials. For example, slow kinetics may impede the formation of well-defined periodic morphologies with long-range order due to the presence of chain entanglements. There are two main (non-excluding) strategies, beyond the typical annealing process, to overcome these difficulties and achieve complex multi-domain morphologies in a cost-effective, robust, and scalable way. The first way is to guide the selfassembly towards the desired state with external means, namely an external field or a patterned substrate.[18, 25, 35] The second approach is based on using highly branched polymers, which have a lower density of entanglements than their linear chain counterparts. To this end, bottle-brush (BB) polymers are very promising as an example of such a highly-branched molecular architecture. 
BB polymers are branched macromolecules with linear side-chains attached onto a linear backbone chain. [30, 31, 33, 37, 38] The molecular architecture of BB polymers can vary, being, for example, densely or loosely grafted, and having flexible or stiff side chains on the backbone (Figure 1). As homopolymers, BB polymers have a significantly lower density of entanglements than their linear chain analogues and exhibit a rich viscoelastic behavior. Due to these unique properties, they are promising candidates for a number of applications, a noteworthy example is their potential in replacing aggrecans in synthetic low friction and high strength synovial joints.[4, 28] As heteropolymers, BB copolymers with distinct type of side chains offer the possibility of tuning the effective molecular interactions, resulting in morphologies that were previously difficult to access with linear chain copolymers. This molecular design path has attracted much attention for applications ranging from photovoltaics and large scale nanopores to nanowires and multi-component nanocapsules.[2, 9, 12, 14, 15, 21, 24, 36, 40, 41, 43] However, the complete morphology map as a function of the BB molecular parameters is still under investigation. $[6,9,10,12,16,32]$

In the classic phase diagram of linear diblock copolymers, the equilibrium morphology is determined by the balance between the competing block interfacial tension and the entropic penalty of stretching the polymer coils to fill the space uniformly.[1] Introducing an asymmetry in the segmental size/stiffness between the blocks, towards rod-coil copolymers, results in significant shifts of the boundaries between morphologies[19, 39] at the cost of slowing down the self-assembly kinetics.[20] These shifts in the boundaries between different ordered phases are of considerable interest, as mechanical, 
rheological, and optical properties of the phases are highly influenced by their topology. Despite significant theoretical advances, the modeling of the selfassembly behavior of di- and multi-block copolymers containing semiflexible units remains an outstanding challenge. $[23,26,34,42]$ In the case of BB diblock copolymers with large aspect ratio, which is equivalent to using thick linear chains, [3] particular effort has been put in the formation of lamellar morphology.[10, 12] However, low aspect ratio BB copolymers may not easily be described by a map on a linear chain architecture, because the "Flory theorem" does not generally extend to polymers having a non-trivial topology.[7] Even for linear chains having small and moderate molecular mass, the molecular conformations deviate from that of flexible coils.[17, 22] It is important to understand how non-trivial molecular architectures, like BB copolymers, can influence the self-assembly towards the desired morphology with the use of a model that includes excluded volume interactions. The authors are not aware of any investigation on the role of the intrinsic backbone chain stiffness in the phase behavior of BB diblock copolymers.

The aim of the current study is to investigate the effect of the backbone intrinsic chain stiffness on the morphologies of BB diblock copolymers having the same volume fractions of the side chains in each block, but different side chain lengths. We use molecular dynamics (MD) simulations of a bead-spring model that incorporates the excluded volume interactions and has allowed the construction of morphology maps[9] of BB diblock copolymers, having a symmetric composition of phase segregating segments and with an apparent backbone chain stiffness influenced by the side chains[38]. We expand these morphology maps by increasing the intrinsic backbone chain stiffness. 
We find a crossover from hexagonally packed cylinders in the case of BB copolymers with flexible backbones to the formation of gyroid/bicontinuous morphologies for stiffer backbones and to lamellar morphologies with asymmetric domain spacing for BB copolymers of even higher stiffness. The latter morphology is counter-intuitive, as an increase of the backbone chain stiffness enhances the anisotropy in molecular shape, which is characteristic for disrupting lamellar morphology in melts of linear chain diblock copolymers. We argue that the above order--order crossover from hexagonally packed cylinders to lamellar morphologies is due to the many-body interactions originating from the backbone stiffness.

\section{Model and methods}

Our simulations were based on a bead-spring model, which was implemented in the large-scale atomic/molecular massively parallel simulator[29] (LAMMPS). Every BB block-copolymer chain contained three types of segments; letters $\mathrm{A}$ and $\mathrm{B}$ denote the different types of beads belonging to the side chains, while $\mathrm{C}$ denotes the backbone segments (Figure 1). We considered compositionally symmetric bottle-brush copolymers, namely the total number of $\mathrm{A}$ and $\mathrm{B}$ segments is the same, i.e., $M_{\mathrm{A}}=M_{\mathrm{B}}=M$, as well as the volume fractions $\left(f_{\mathrm{A}}=f_{\mathrm{B}}\right)$. Moreover, grafted side chains of the same type always have the same length. Hence, by varying the length of the side chains $\left(N_{\mathrm{A}}\right.$ and $\left.N_{\mathrm{B}}\right)$, the total number of backbone segments varied accordingly since, in our case, every side chain was attached to a single backbone segment. Thus, the volume fraction of the backbone segments, $f_{\mathrm{C}}$, was not fixed with variation of the side chain length, $f_{\mathrm{C}}=1-f_{\mathrm{A}}-f_{\mathrm{B}}=1-2 f_{\mathrm{A}}=1-2 f_{\mathrm{B}}$. Hence, 
the obtained molecular architectures ranged from linear triblock copolymers (when $N_{\mathrm{A}}=N_{\mathrm{B}}=M$ ) to BB copolymers where each backbone segment was connected to one A- or B-type segments, (cf. Figure 1).

The BB diblock copolymers were placed in a cubic simulation box with periodic boundary conditions applied in all directions. The systems are equilibrated at NPT statistical ensemble (constant number of particles, constant temperature, and constant pressure), where the chosen pressure $P$ corresponding to the ambient pressure is applied isotropically, and the temperature of the system was such to enable microphase separation between A- and B-type segments for given interaction parameters. Moreover, the phase separation was driven by the incompatibility between A- and B-type segments, as none of the A- or B-type segments has an energetic preference for the backbone segments (details of the model can be found in Ref. 9). The map between the degree of segregation and our bead-spring model, which is expressed by the Flory-Huggins parameter $\chi$,[11] has been discussed recently.[8] In our study, the $\chi$ parameter for A- and B-type segments was between 16 and 40 depending on the molecular weight, which corresponds to the intermediate segregation regime.[1] In this regime, we were able to obtain well-defined morphologies far from the strong segregation regime and the order-disorder transition. The stiffness along the backbone chain was modeled by the harmonic angle potential, $V(\theta)=\kappa\left(\theta-\theta_{0}\right)^{2}$, where $\kappa$ describes the degree of stiffness and $\theta_{0}$ the equilibrium angle; in our study we set $\theta_{0}=180^{\circ}$. Within the intermediate segregation regime, the thermal fluctuations had little qualitative impact on the stiffness and the final morphology. A schematic illustration of $\mathrm{BB}$ diblock copolymer molecular conformation at different values 
of $\kappa$ is presented schematically in Figure 1.

\section{Results and discussion}

We constructed morphology maps based on the variation of the molecular mass and two molecular parameters, namely $\kappa$, which is the backbone stiffness, and the length of the side chains for one of the blocks $\left(N_{\mathrm{A}}\right.$ or $\left.N_{\mathrm{B}}\right)$. The latter choices were based on the following two observations. Firstly, we have shown that for $\kappa=0$ hexagonally packed cylinders are formed when there is significant difference between the length of side chains of each block even though for the volume fractions of the two blocks $f_{\mathrm{A}}=f_{\mathrm{B}}$; for all other combinations of side chains lengths of the two blocks $N_{\mathrm{A}}$ and $N_{\mathrm{B}}$ lamellar morphologies were observed (Figure 2).[9] Secondly, the lamellae forming systems obtained for $\kappa=0$ with our model do not undergo morphology changes with an increase in $\kappa$, Figure 3a. As we discuss below, the cylinder-forming systems $(\kappa=0)$ exhibit a rich morphology behavior with variation of the stiffness $(\kappa>0)$. Hence, our parameter space is defined by the backbone stiffness $\kappa$ and the side chain length of one of the two blocks $N_{\mathrm{A}}$.

The obtained morphology maps for different $M$ (the total number of A or B segments) are presented in Figure 3c. Clearly, a small degree of intrinsic stiffness along the backbone chain (e.g., $0<\kappa \leq 0.5$ ) does not influence the expected final morphology. Moreover, in the case of $N_{\mathrm{A}}>M / 4$, where the length of the side chains between the two blocks becomes similar in length, we do not observe any changes in the final morphology for all degrees of backbone stiffness that we have explored here. However, we find

significant morphology changes when the side chains are highly asymmetric 
in length and $\kappa>0$. In particular, there is a crossover from hexagonally packed cylinders (the expected morphology for $\kappa=0[9]$ ) to a bicontinuous morphology for $0.5 \leq \kappa \leq 2$ for $N_{\mathrm{A}}=1$ and $N_{\mathrm{B}}=M$ (Figure 3b, c). For a stiffer backbone chain, $\kappa \geq 2$, a two-domain lamellar morphology having two distinct domain length scales $\left(d_{\mathrm{A}}\right.$ and $\left.d_{\mathrm{B}}\right)$ is formed (Figure $3 \mathrm{~b}$ ). The difference in the size of the two domains $\left(d_{\mathrm{A}}\right.$ and $\left.d_{\mathrm{B}}\right)$ can be tuned with variation of $N_{\mathrm{A}}$ (cf. Figure $3 \mathrm{~b}$ the cases of $M=16$ ). $M$ plays an important role, as, for larger molecular mass BB diblock copolymers, a higher degree of asymmetry in the length of side chains between the two blocks can be achieved. From the perspective of realizing applications these features seem very attractive, because the formation of lamellar morphology having highly asymmetric spacing with linear chain diblock copolymer systems without additives is particularly challenging.

An increase in the stiffness along the backbone leads to an anisotropic molecular shape, i.e., an increase in the aspect ratio. This effect becomes more pronounced as the molecular mass increases resulting in larger asymmetry between the two blocks with variation of the side chain length. At this point, we wonder: "Why does an increase in the anisotropy of the molecular shape lead to a disruption of the cylindrical symmetry and the eventual formation of a lamellar morphology?". To probe this question we calculated the order parameter $S=P_{2}(\cos \theta)=\left\langle\left(3 \cos ^{2} \theta-1\right) / 2\right\rangle$ for the end-to-end vector along the $\mathrm{BB}$ backbone, where angle brackets indicate an ensemble average over all $\mathrm{BB}$ copolymers and $P_{2}$ is the Legendre polynomial of second order (Figure 4a).[5] The order parameter $S$ takes values close to -0.5 when backbones are oriented perpendicular to each other, while values close to 1 
indicate that the backbones orient parallel with each other. The dependence of the order parameter with backbone stiffness is non-trivial. Small values of $\kappa$ lead to a small increase in $S$, while the BB form hexagonally packed cylinders. This increase is mainly driven by BB stacking radially on a plane perpendicular to the cylinders. However, this type of stacking is not stable for $\kappa>0.6$ and bicontinuous morphology is observed, causing $S$ to drop since now BB molecules orient is all directions. For $\kappa>2$, BBs form lamellar morphologies resulting in a nematic-like order of the backbones. We rationalize this effect as a significant increase in $\kappa$ effectively transforming one of the blocks into a rod-like polymer. We note that between rod-like molecules there are many-body interactions, which drive the packing the rod-like molecules together with a significant orientational ordering; [20] in our case this molecular orientation takes the form of nematic-like order between the neighboring BB diblock copolymers. We argue that this effect disrupts the cylindrical symmetry in the packing of $\mathrm{BB}$ copolymers resulting in a change in the final morphology, as described schematically in Figure $4 \mathrm{~b}$.

In rod-coil copolymer systems, the rod-like block has a propensity to crystallize, thus inhibiting the self-assembly kinetics.[20] In our case, we observe a high orientational order as $\kappa$ increases and $M$ decreases. Although, high orientational order is associated with slowing down of the dynamics, our system do not freeze due to the presence of the side chains, which frustrate the path towards crystalization. In other words, increasing $\kappa$ and reducing $M$ results in high degree of orientational order while the system remains in the liquid state. We then expect that the kinetics of the morphology formation becomes slower as $\kappa$ increases, but at the same time we expect faster kinetics 
from BB stiff copolymers with respect to equivalent linear chain copolymers, namely the fast kinetics due to the low density of chain entanglements. Overall, our findings suggest that the intrinsic backbone stiffness is an important factor in the self-assembly kinetics and in the final morphology of BB diblock copolymer systems.

\section{Conclusions}

In summary, we investigated the role of the backbone stiffness in the selfassembly of BB diblock copolymers. By using MD simulations of a coarsegrained model, we have constructed morphology maps that capture how an increase in the intrinsic backbone chain stiffness results in an order-order crossover from hexagonally-packed cylinders to a lamellar morphology with highly asymmetric domain spacing. This behavior is unexpected given that the increase of backbone stiffness leads to an increased anisotropy of the overall molecular shape. The preference of a lamellar morphology originates from the effective many-body interactions between the rod-like blocks that favors a nematic-like packing for one of the blocks. This type of packing disrupts the cylindrical symmetry resulting in lamellar morphologies of highly asymmetric domain spacings. We anticipate that our work will stimulate further investigation in the direction of considering the effect of backbone chain stiffness in the morphology behavior of block copolymer systems.

\section{Acknowledgement}

Dr. A. Chremos gratefully acknowledges the support of the NIST Director's Office through the NIST Fellows' postdoctoral grants program. Official 
contribution of the U.S. National Institute of Standards and Technology not subject to copyright in the United States.

\section{References}

[1] F.S. Bates, Science 251 (1991) 898-905.

[2] J. Bolton, T.S. Bailey, J. Rzayev, Nano Lett. 11 (2011) 998-1001.

[3] Z. Cao, J.M.Y. Carrillo, S.S. Sheiko, A.V. Dobrynin, Macromolecules 48 (2015) 5006-15.

[4] J.M. Carrillo, W.M. Brown, A.V. Dobrynin, Macromolecules 45 (2012) 8880-91.

[5] P.M. Chaikin, T.C. Lubensky, Principles of Condensed Matter Physics, Cambridge University Press, Cambridge, 2000.

[6] Y. Choo, L.H. Mahajan, M. Gopinadhan, D. Ndaya, P. Deshmukh, R.M. Kasi, C.O. Osuji, Phase behavior of polylactide-based liquid crystalline brushlike block copolymers, Macromolecules 48 (2015) 8315-22.

[7] A. Chremos, J.F. Douglas, J. Chem. Phys. 143 (2015) 111104.

[8] A. Chremos, A. Nikoubashman, A.Z. Panagiotopoulos, J. Chem. Phys. 140 (2014) 054909.

[9] A. Chremos, P. Theodorakis, ACS Macro Lett. 3 (2014) 1096-100.

[10] S.J. Dalsin, T.G. Rions-Maehren, M.D. Beam, F.S. Bates, M.A. Hillmyer, M.W. Matsen, Bottlebrush block copolymers: quantitative theory and experiments, ACS Nano (2015). 10.1021/acsnano.5b05473. 
[11] P.J. Flory, Principles of Polymer Chemistry, Cornell University Press, Ithaca, NY, 1953.

[12] W. Gu, J. Huh, S.W. Hong, B.R. Sveinbjornsson, C. Park, R.H. Grubbs, T.P. Russell, ACS Nano 7 (2013) 2551-8.

[13] I.W. Hamley, Angewandte Chemie International Edition 42 (2003) 15.

[14] W. Han, M. Byun, L. Zhao, J. Rzayevc, Z. Lin, J. Mater. Chem. 21 (2011) 14248.

[15] S. Hanski, N. Houbenov, J. Ruokolainen, D. Chondronicola, H. Iatrou, N. Hadjichristidis, O. Ikkala, Hierarchical ionic self-assembly of rodcomb block copolypeptide-surfactant complexes, Biomacromolecules 7 (2006) 3379-84.

[16] S.W. Hong, W. Gu, J. Huh, B.R. Sveinbjornsson, G. Jeong, R.H. Grubbs, T.P. Russell, ACS Nano 7 (2013) 9684-92.

[17] C. Jeong, J.F. Douglas, J. Chem. Phys. 143 (2015) 144905.

[18] S.O. Kim, H.H. Solak, M.P. Stoykovich, N.J. Ferrier, J.J. de Pablo, P.F. Nealey, Nature 424 (2003) 411-4.

[19] N.A. Kumar, V. Ganesan, J. Chem. Phys. 136 (2012) 101101.

[20] M. Lee, B.K. Cho, W.C. Zin, Chem. Rev. 101 (2001) 3869-92.

[21] F. Liu, J. Hu, G. Liu, C. Hou, S. Lin, H. Zou, G. Zhang, J. Sun, H. Luo, Y. Tu, Macromolecules 46 (2013) 2646-57. 
[22] M.L. Mansfield, A. Tsortos, J.F. Douglas, J. Chem. Phys. 143 (2015) 124903.

[23] M.W. Matsen, J. Chem. Phys. 104 (1996) 7758-64.

[24] G.M. Miyake, V.A. Piunova, R.A. Weitekamp, R.H. Grubbs, Angew. Chem. Int. Ed. 51 (2012) 11246-8.

[25] A. Nikoubashman, R.A. Register, A.Z. Panagiotopoulos, Soft Matter 9 (2013) 9960-71.

[26] B.D. Olsen, M. Shah, V. Ganesan, R.A. Segalman, Macromolecules 41 (2008) 6809.

[27] C. Parka, J. Yoonb, E.L. Thomas, Polymer 44 (2012) 6725-60.

[28] T. Pettersson, A. Naderi, R. Makuska, P.M. Claesson, Langmuir 24 (2008) 3363-47.

[29] S.J. Plimpton, J. Comput. Phys. 117 (1995) 1.

[30] S. Rathgeber, T. Pakula, A. Wilk, K. Matyjaszewski, K.L. Beers, J. Chem. Phys. 122 (2005) 124904.

[31] S. Rathgeber, T. Pakula, A. Wilk, K. Matyjaszewski, H. Lee, K.L. Beers, Polymer 47 (2006) 7318-27.

[32] J. Rzayev, ACS Macro Lett. 1 (2012) 1146-9.

[33] S.S. Sheiko, B.S. Sumerlin, K. Matyjaszewski, Cylindrical molecular brushes: Synthesis, characterization, and properties, Progress in Polymer Science 33 (2008) 759-85. 
[34] W.D. Song, P. Tang, F. Qiu, Y. Yang, A.C. Shi, J. Chem. Phys. B 115 (2011) 8390.

[35] M.P. Stoykovich, H. Kang, K.C. Daoulas, G. Liu, C.C. Liu, J.J. de Pablo, M. Muller, P.F. Nealey, ACS NANO 1 (2007) 168-75.

[36] B.R. Sveinbjornsson, R.A. Weitekamp, G.M. Miyake, Y. Xia, H.A. Atwater, R.H. Grubbs, Proc. Natl. Acad. Sci. U.S.A. 109 (2012) 14332.

[37] P.E. Theodorakis, H.P. Hsu, W. Paul, K. Binder, J. Chem. Phys. 135 (2011) 164903.

[38] P.E. Theodorakis, W. Paul, K. Binder, Macromolecules 43 (2010) 513748.

[39] J.D. Vavasour, M.D. Whitmore, Macromolecules 26 (1993) 7070-5.

[40] R. Verduzco, X. Li, S.L. Peseka, G.E. Stein, Chem. Soc. Rev. 44 (2015) 2405-20.

[41] Y. Xia, B.D. Olsen, J.A. Kornfield, R.H. Grubbs, Efficient Synthesis of Narrowly Dispersed Brush Copolymers and Study of Their Assemblies: The Importance of Side Chain Arrangement, J. Am. Chem. Soc. 131 (2009) 18525-32.

[42] G.A. Yang, P. Tang, Y.L. Yang, Q.A. Wang, J. Chem. Phys. B 114 (2010) 14897.

[43] M. Zhang, A.H.E. Müller, Cylindrical polymer brushes, J. Polym. Sci. Part A: Polym. Chem. 43 (2005) 3461-81. 


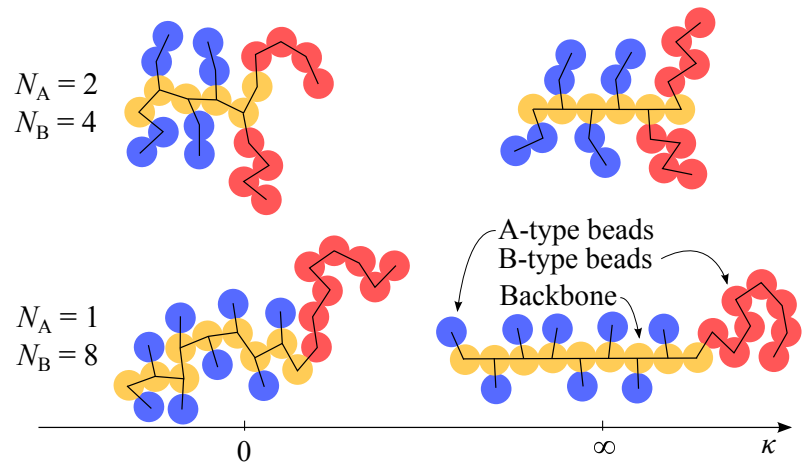

Figure 1: Effect of the backbone stiffness $\kappa$ on each BB copolymers for different combinations of side chain lengths $N_{\mathrm{A}}$ and $N_{\mathrm{B}}$. BB copolymers have the same volume fractions of phase segragated type-A and and type-B segments. Different colors correspond to different type of beads as indicated.

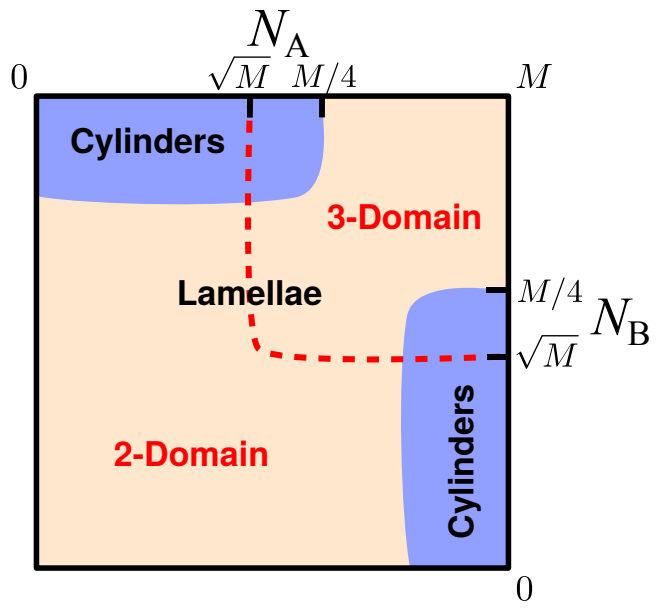

Figure 2: Morphology diagram of BB copolymers with flexible backbones $(\kappa=0) . N_{\mathrm{A}}$ and $N_{\mathrm{B}}$ correspond to the side chain lengths of the type-A and the type-B blocks, respectively. Approximate boundaries are drawn based on the results obtained in Ref. 9 for $M>16$. 

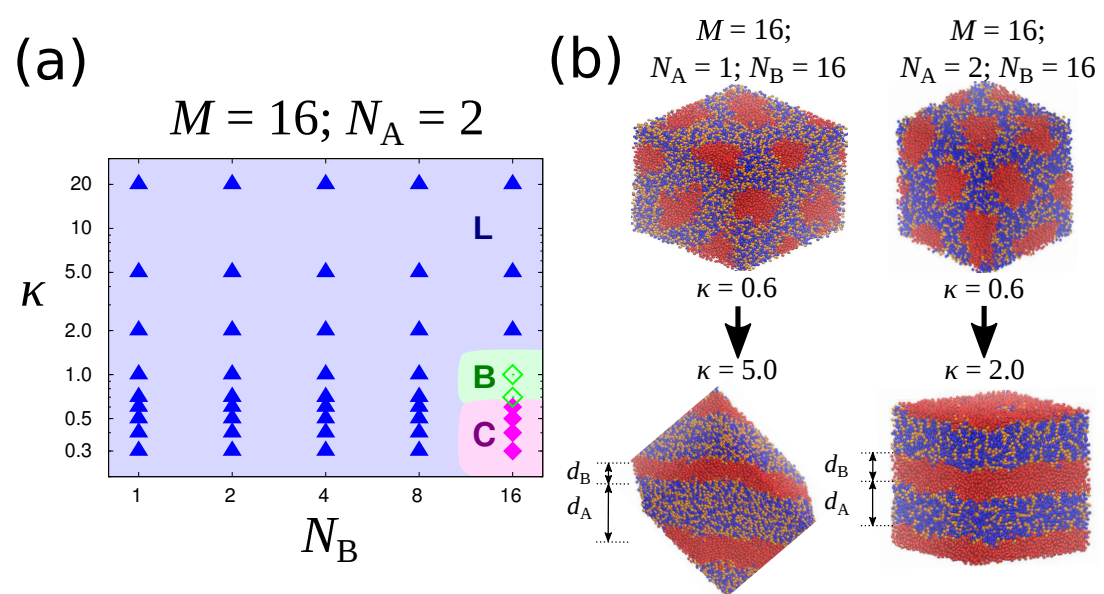

$$
\begin{array}{cc}
M=32 ; & M=16 \\
N_{\mathrm{A}}=1 ; N_{\mathrm{B}}=32 & N_{\mathrm{A}}=4 ; N_{\mathrm{B}}=16
\end{array}
$$

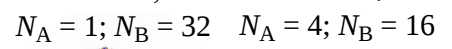

(c) $\quad M=8 ; N_{\mathrm{B}}=8$
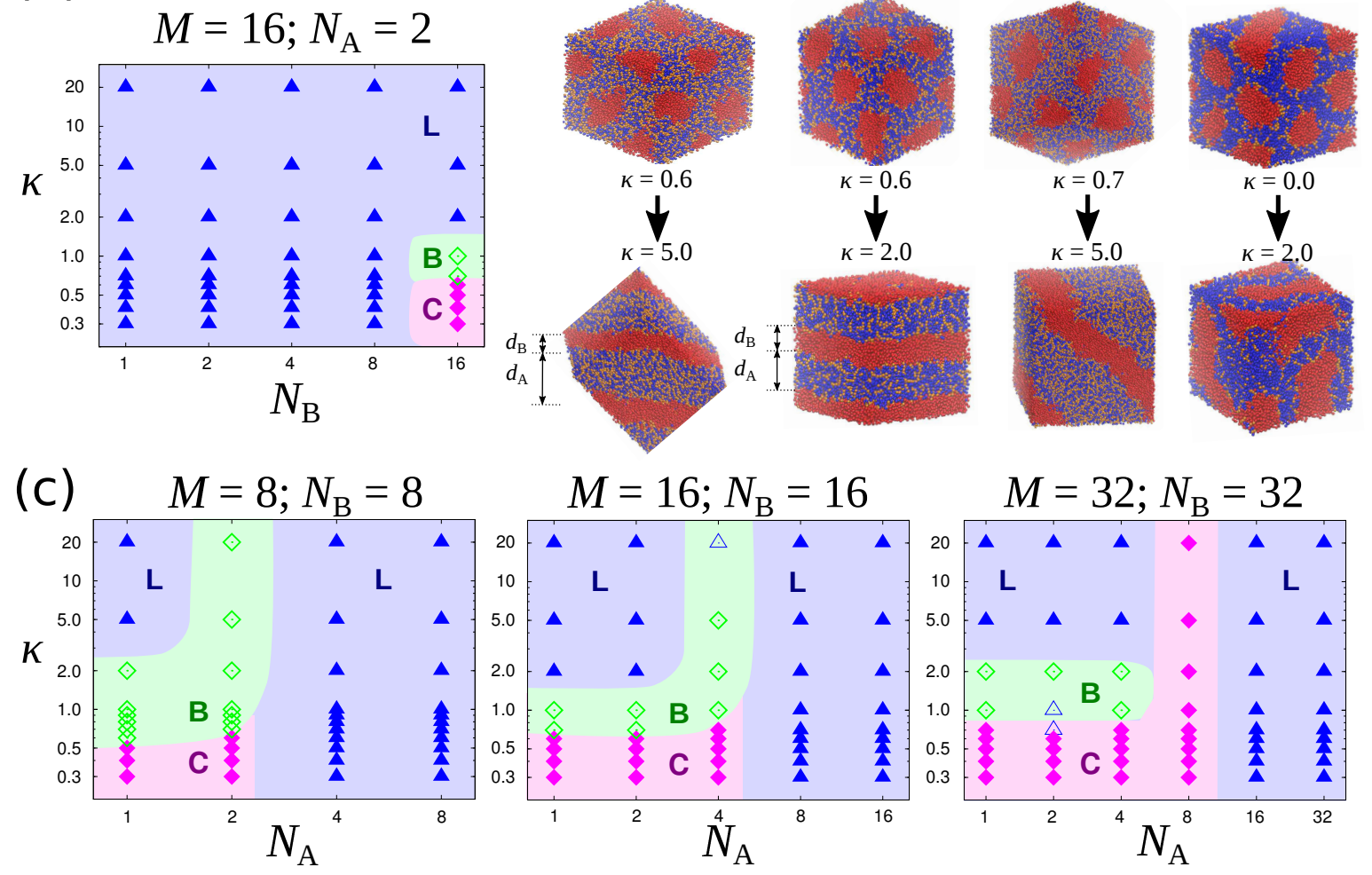

Figure 3: (a) Morphology diagram for $M=16$ of BB diblock copolymers with $N_{\mathrm{A}}=2$ as function of $N_{\mathrm{B}}$ and $\kappa$. (b) Snapshots illustrating crossovers from cylinders to lamellar or bicontinuous morphologies for different cases as indicated. (c) Morphology diagrams for $M=8$ (left), 16 (center), and 32 (right) of bottle-brush diblock copolymers based on the variation of the backbone stiffness, $\kappa$, and the length of the B-block side chains $N_{\mathrm{B}}=M$. The symbols correspond to lamellae (triangles), cylinders (fill diamonds), and bicontinuous (open diamonds), and metastable (open triangles). 

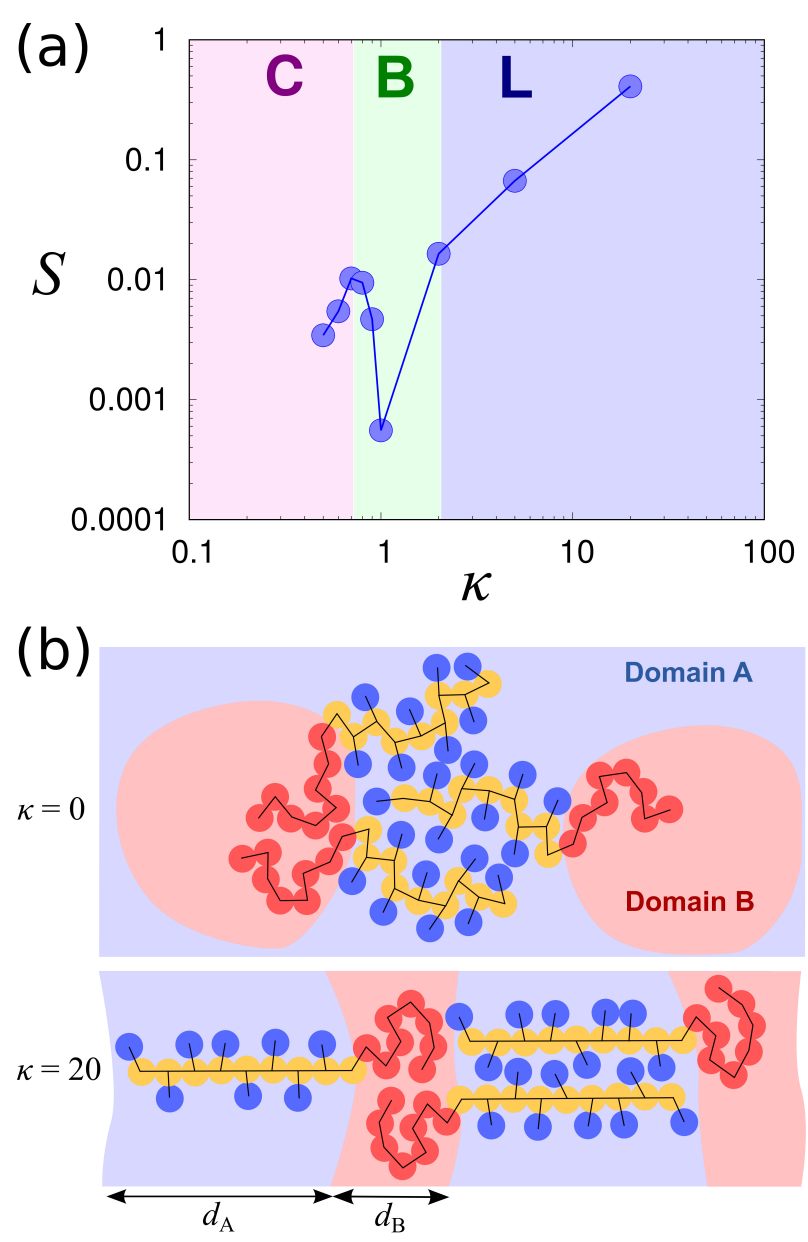

Figure 4: (a) The order parameter $S$ as a function of the stiffness parameter $\kappa$ for a system with $M=8, N_{A}=1$, and $N_{B}=8$. The labels, C, B, and L indicate the range of $\kappa$ for cylinder, bicontinuous, and lamellar morphologies, respectively. (b) Schematic description for the packing of BB diblock copolymers having $M=8$ for $\kappa=0$ (flexible backbone) and for $\kappa=20$ (very stiff backbone), as well as the resulting morphology due to this packing. 